\title{
Surface Characterisation of a Ferroelectric Single Crystal by Kelvin Probe Force Microscopy
}

\author{
Kenny Lau ${ }^{1}$, Yun Liu ${ }^{1 *}$, Qian $\mathrm{Li}^{1}$, Zhenrong $\mathrm{Li}^{2}$, Ray L. Withers ${ }^{1}$, Zhuo $\mathrm{Xu}^{2}$ \\ ${ }^{1}$ Research School of Chemistry, The Australian National University, Canberra, Australia; ${ }^{2}$ Electronic Materials Research Laboratory, \\ Xi'an Jiaotong University, Xi'an, China. \\ Email: *yliu@rsc.anu.edu.au
}

Received May $10^{\text {th }}, 2013$; revised June $11^{\text {th }}, 2013$; accepted June $30^{\text {th }}, 2013$

Copyright (C) 2013 Kenny Lau et al. This is an open access article distributed under the Creative Commons Attribution License, which permits unrestricted use, distribution, and reproduction in any medium, provided the original work is properly cited.

\begin{abstract}
We investigated the surface potential dynamics of a ferroelectric $\mathrm{Pb}\left(\mathrm{In}_{1 / 2} \mathrm{Nb}_{1 / 2}\right) \mathrm{O}_{3}-\mathrm{Pb}\left(\mathrm{Mg}_{1 / 3} \mathrm{Nb}_{2 / 3}\right) \mathrm{O}_{3}-\mathrm{PbTiO}_{3}(\mathrm{PIMNT})$ single crystal using Kelvin probe force microscopy (KPFM). The initial surface potential is a function of the applied bias since it reflects the interplay between the polarisation and screen charges. It is suggested that the different rates of tip injected charges are responsible for the asymmetric behaviour of the initial surface potential dependent on the sign of the applied bias. The polarisation, screen and tip injected charges are considered to explain the difference in surface potential dynamics.
\end{abstract}

Keywords: Surface Potential; KPFM; Ferroelectric

\section{Introduction}

The introduction of the atomic force microscope (AFM) was of fundamental importance to surface characterisation techniques because it allowed probing of the force between an atomically sharp tip and a sample surface [1]. In early experiments, local mechanical and adhesive forces were determined by studying the van der Waal's and electrostatic tip-surface interaction. This allowed the correlation between surface topography and properties at the nanoscale [2]. It was quickly realized that the scope of AFM imaging could be expanded by modifying the tip-surface interaction forces [3]. This insight led to the use of a conductive tip and the development of a range of electrical measurement techniques by biasing the tip with respect to the sample [2,3]. Depending on the operation mode and the response measured this gave rise to electric force microscopy, piezoresponse force microscopy (PF$\mathrm{M})$, scanning conductance microscopy and Kelvin probe force microscopy (KPFM) - to name but a few techniques [4].

In KPFM, following the recording of the surface topography the tip is lifted a fixed distance above the surface in order to maintain a constant van der Waal's interaction force [5]. During the lifted scan, a sinusoidal signal with a dc offset is applied to the AFM tip

\footnotetext{
${ }^{*}$ Corresponding author.
}

$$
V_{\text {tip }}=V_{D C}+V_{A C} \sin (\omega t)
$$

This results in an electrostatic force:

$$
F=\frac{1}{2} \frac{d C}{d z}(\Delta \mathrm{V})^{2}
$$

where $d C /$ dzis is the tip surface capacitance gradient and is the potential difference between the tip and the surface (i.e. $\Delta \mathrm{V}=\mathrm{V}_{\text {tip }}-\mathrm{V}_{\mathrm{SP}}$ ) [5]. Due to the $\mathrm{AC}$ voltage applied to the tip, the electrostatic force is the sum of a static component, a first harmonic component at $1 \omega$ and a second harmonic component at $2 \omega$ :

$$
\begin{gathered}
F_{D C}=\frac{1}{2} \frac{d C}{d z}\left(\left(V_{D C}-V_{S P}\right)^{2}+\frac{V_{A C}^{2}}{2}\right) \\
F_{1 w}=\frac{d C}{d z}\left(\left(V_{D C}-V_{S P}\right) V_{A C} \sin \sin (\omega t)\right) \\
F_{2 w}=-\frac{1}{4} \frac{d C}{d z}\left(V_{A C}^{2} \cos \cos (2 \omega t)\right)
\end{gathered}
$$

A lock-in amplifier is used to extract the electrical force at the first harmonic $\left(F_{\omega}\right)$. The surface potential is then obtained with a feedback loop that adjusts the applied dc bias to minimize $\mathrm{V}_{D C}-\mathrm{V}_{\mathrm{SP}} \quad[3,5]$. Hence the surface potential is obtained.

KPFM has been successfully employed to study a ran- 
ge of systems [5] including metals [2,5], semiconductors [6,7], organic thin films [8] and DNA [9]. In this paper, we focus on using KPFM for the characterisation of the surface potential of a ferroelectric single crystal. In a ferroelectric, the termination of the spontaneous polarisation at the surface results in polarisation bound charges [10-12]. To achieve a thermodynamically stable state, screen charges are necessary for charge balance. The interplay between the polarisation and these screen charges gives rise to the surface potential $[10,13,14]$.

The surface potential of ferroelectrics is a candidate for memory devices $[15,16]$. Previous studies on ferroelectric thin films have concluded that the surface potential depends on the applied bias [15-19]. However, an asymmetry in the initial surface potential, dependent on the sign of the applied bias, is observed $[17,18]$. This is typically attributed to the presence of an internal electric field at the ferroelectric-electrode interface that either enhances or opposes the applied bias $[17,18]$. Studies on the surface potential relaxation of ferroelectric polycrystalline films are complicated by the presence of potential pits formed at grain boundaries [15] and the impact of grain size on electronic properties [20]. In this study, we report on the surface potential dynamics of the relaxor ferroelectric $\mathrm{Pb}\left(\mathrm{In}_{1 / 2} \mathrm{Nb}_{1 / 2}\right) \mathrm{O}_{3}-\mathrm{Pb}\left(\mathrm{Mg}_{1 / 3} \mathrm{Nb}_{2 / 3}\right) \mathrm{O}_{3}-\mathrm{PbTiO}_{3}$ (PIMNT). A bulk single crystal is used to eliminate grain related effects and the effect of an internal electric field at the ferroelectric-electrode interface.

\section{Experimental}

An (001) cut $0.25 \mathrm{~Pb}\left(\operatorname{In}_{1 / 2} \mathrm{Nb}_{1 / 2}\right) \mathrm{O}_{3}-0.44 \mathrm{~Pb}\left(\mathrm{Mg}_{1 / 3} \mathrm{Nb}_{2 / 3}\right)$ $\mathrm{O}_{3}-0.31 \mathrm{PbTiO}_{3}$ single crystal was optically polished to a thickness of $\sim 0.7 \mathrm{~mm}$. The crystal was mounted on an electrically grounded metal substrate with conductive silver paste (Ted Pella). KPFM measurements were performed on a commercial atomic force microscope (Cypher, Asylum Research) under ambient conditions with Pt-coated Si conductive probes (Olympus AC240TM, force constant $\sim 2 \mathrm{~N} / \mathrm{m}$ and tip radius $\sim 28 \mathrm{~nm}$ ). PFM was utilised to confirm the ferroelectric polarization state of the single crystal before and after poling.

A voltage bias was applied to four 2 by $2 \mu \mathrm{m}$ squares in contact mode. The voltage bias, applied to the tip, was between -10 and $-40 \mathrm{~V}$. The surface potential was recorded immediately after the bias was applied for up to 75 minutes. KPFM scans were acquired at $2 \mathrm{~Hz}$ with a lift height of $30 \mathrm{~nm}$. The surface potential of a particular bias was taken as the average surface potential value over the corresponding 2 by $2 \mu \mathrm{m}$ square. This process was repeated three times and the results were averaged. A similar scheme was used for positive voltage biases.

\section{Results and Discussion}

A typical PFM phase image of (001)-oriented PIMNT single crystals is shown in Figure 1(a). The contrast in the PFM phase image arises from whether the spontaneous polarisation is directed towards the surface or away from it. The labyrinthine polarisation domain pattern is characteristic of relaxor ferroelectrics [21]. The surface potential of the as-grown crystal is zero (not shown) as expected for an energetically stable state. The PFM phase image collected 75 minutes after application of a voltage bias is shown in Figure 1(b). The application of the bias has resulted in reorientation of the polarisation. This is because the applied bias exceeds the coercive voltage of PIMNT ( 4 V) [22]. The surface potential immediately after application of a voltage bias is shown in Figure 1(c). The value of the applied bias is shown in white text. Note that the surface potential increases with increasing applied bias and tends towards the square bias pattern. KPFM images were continuously collected over a period of 75 minutes. After this time, the surface potential had dropped considerably (Figure 1(d)). Only the $-40 \mathrm{~V}$ bias square retained its shape. A typical surface potential as a function of time when a negative bias is applied is shown in Figure 2(a). To quantify the decay of the surface potential, an exponential decay model was used

$$
V_{S P}=V_{S P 0}+\operatorname{Aexp}\left(-\frac{t}{\tau}\right)
$$

where $A$ is a prefactor and $\tau$ is the relaxation time [11]. From this model, the initial surface potential $\left(V_{\mathrm{SP}}+A\right)$ and half-life $t_{\frac{1}{2}}=-\tau \ln \left(\frac{1}{2}-\frac{V_{S P 0}}{2 A}\right)$ were derived and plotted in Figures 2(b) and (c)), respectively.
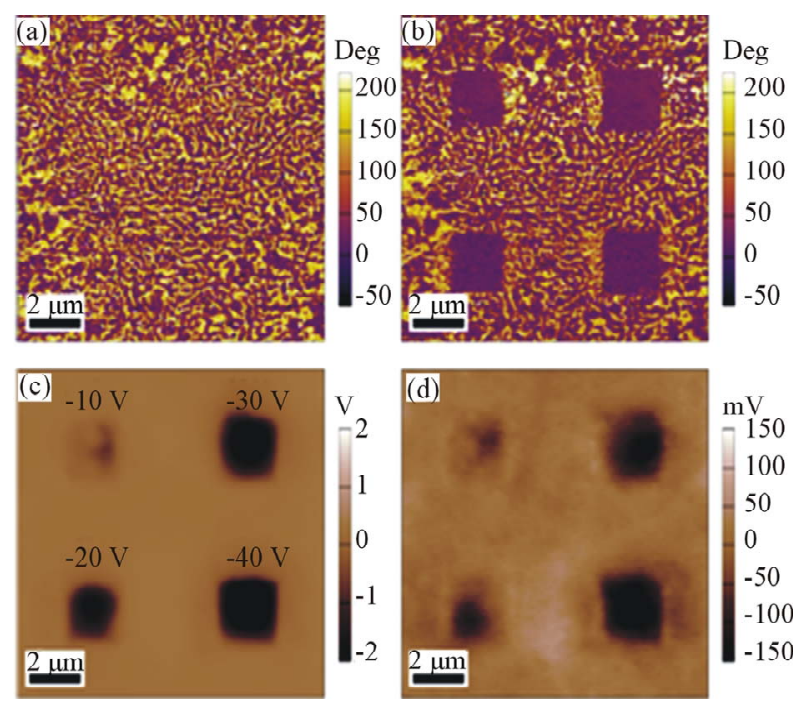

Figure 1. PFM phase image of (001) oriented PIMNT (a) before and (b) 75 minutes after application of voltage bias. Surface potential image obtained (c) immediately after and (d) 75 minutes after application of voltage bias. The value of the applied bias is shown in white (c). 


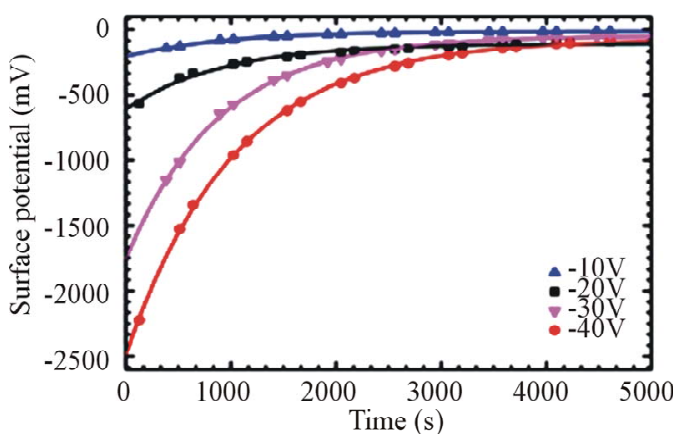

(a)

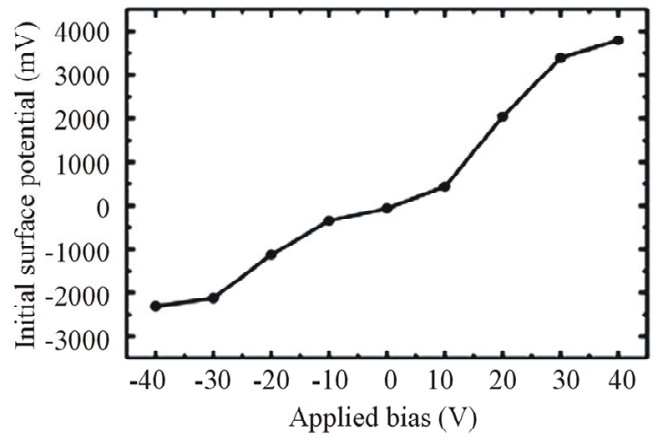

(b)

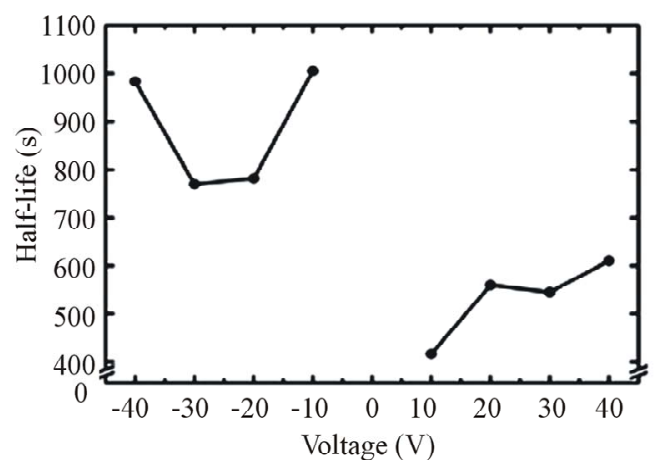

(c)

Figure 2. (a) Typical surface potential as function of time for different applied biases. Experimental data are shown as symbols while the line is the exponential fit. The (b) initial surface potential and (c) half-life as a function of applied bias was extracted from the exponential fit. The lines are a guide for the eye.

After a bias is applied, the polarisation is oriented parallel to the applied field. A positive (negative) bias causes the polarisation to point away from (towards) the surface resulting in negative (positive) polarisation charges. The negative (positive) polarisation charges are compensated by positive (negative) screen charges. Screen charges can be internal, originating from defects, mobile carriers or tip injected charges, or external coming from the ambient environment [16]. Thus, the sign of the surface potential is determined by the sign of the screen charge $[10,15]$, which is identical to the sign of the applied bias (Figure 3). As the magnitude of the applied bias is in-

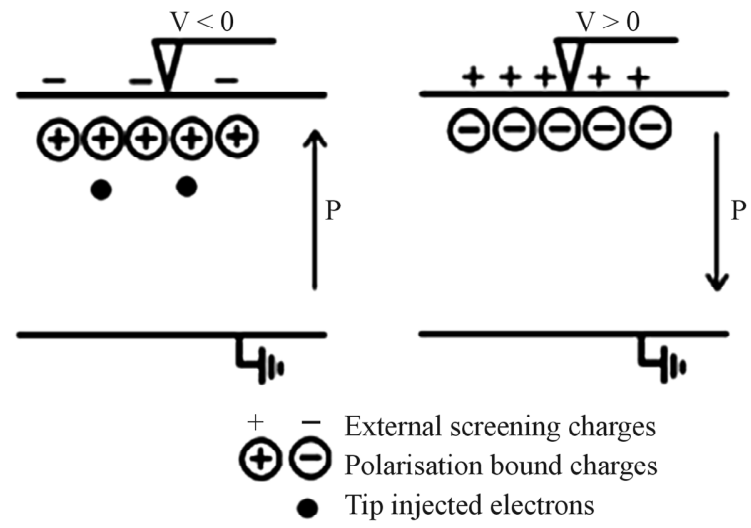

(a)

(b)

Figure 3. Schematic diagrams of polarisation charges, tip injected electrons and external screen charges when a (a) negative bias or (b) positive bias is applied. The polarisation is parallel to the applied field.

creased, the polarisation charge increases and the concomittant screen charge increases leading to an increase in the magnitude of the surface potential. This relationship is nonlinear presumably due to the saturation of dipoles aligned parallel to the field and Coulombic repulsion between the surface charges [19]. The magnitude of the surface potential after applying a positive bias is greater than after applying a negative bias. In ferroelectric thin films, this asymmetry in the initial surface potential is ascribed to an internal electric field at the ferroelectric-electrode interface $[17,18]$. The internal electric field is independent of the applied bias and is always aligned in a particular direction. If the internal field is directed away from the surface, the applied field is enhanced when a positive bias is applied leading to more polarisation charges and consequently more screen charges and a higher surface potential. Conversely, the internal field opposes the applied field when a negative bias is used resulting in a lower surface potential [17]. However, for the thick single crystal PIMNT used in this study, this explanation is inadequate for, even if an internal field were present at the ferroelectric-electrode interface, the thickness of the sample would render its effect negligible. Instead, the asymmetry in the initial surface potential is rationalized by consideration of the charge dynamics present at the ferroelectric surface. As shown in Figure 3, since the ferroelectric surface is charge neutral, the sum of polarisation charges and screen charges is zero. The asymmetry is likely to be due to the different rates of tip injected charges. In particular, the barrier for hole injection is greater than for electron injection [23]. Therefore, when a negative bias is applied, electron injection occurs reducing the external screen charges (Figure 3(a)). Since this does not occur when a positive bias is applied, more screen charges are required leading to the observed asymmetry in the initial surface potential (Figure 3(b)). 
While the precise behaviour of the surface potential half-life is not readily explained, the preceding explanation based on the surface charges at the ferroelectric surface is adequate to explain the general trend that the surface potential half-life after a negative bias is applied is greater than when a positive bias is applied. As the polarisation relaxes, there is a reduction of polarisation charges [24]. For a negative biased region, this is accompanied either by a reduction in tip injected electrons or external screen charge. However, in a positive biased region, the only mechanism is the reduction of external screen charge hence the surface potential life-time of positive biased regions is lower than that of negative biased regions. Moreover, the higher initial surface potential in the positive biased region may be responsible for its shorter half-time due to stronger Coulombic repulsion forces [17].

\section{Conclusion}

KPFM is a versatile technique that allows the local characterisation of the surface potential for a variety of materials. We used KPFM to determine the surface potential dynamics after the application of a bias on a ferroelectric PIMNT single crystal. The dependence of the initial surface potential on the applied bias, the asymmetry of the initial surface potential and the half-life of the surface potential are explained by considering the dynamics of the polarisation, screen and tip injected charges present.

\section{Acknowledgements}

K. Lau, Y. Liu, Q. Li and R. L. Withers acknowledge financial support from the Australian Research Council (ARC) in the form of ARC Discovery Project Grants. Y. Liu is the recipient of an Australian Research Council Future Fellowship.

\section{REFERENCES}

[1] S. Magonov, J. Alexander and S. Wu, "Advancing Characterization of Materials with Atomic Force Microscopy-Based Electric Techniques," In: S. V. Kalinin and A. Gruverman, Ed., Scanning Probe Microscopy of Functional Materials, Springer, New York, 2011, pp. 233-300.

[2] R. Berger, H. J. Butt, M. B. Retschke and S. A. L. Weber, "Electrical Modes in Scanning Probe Microscopy," Macromolecular Rapid Communications, Vol. 30, No. 14, 2009, pp. 1167-1178. doi:10.1002/marc. 200900220

[3] A. Avila and B. Bhushan, "Electrical Measurement Techniques in Atomic Force Microscopy," Critical Reviews in Solid State and Materials Sciences, Vol. 35, No. 1, 2010, pp. 38-51. doi:10.1080/10408430903362230

[4] S. V. Kalinin, S. Jesse, B. J. Rodriguez, K. Seal, A. P. Baddorf, T. Zhao, Y. H. Chu, R. Ramesh, E. A. Eliseev, A. N. Morozovska, B. Mirman and E. Karapetian, "Re- cent Advances in Electromechanical Imaging on the Nanometer Scale: Polarization Dynamics in Ferroelectrics, Biopolymers, and Liquid Imaging," Japanese Journal of Applied Physics, Vol. 46, No. 9A, 2007, pp. 5674-5685.

[5] W. Melitz, J. Shen, A. C. Kummel and S. Lee, "Kelvin Probe Force Microscopy and Its Application," Surface Science Reports, Vol. 66, No. 1, 2011, pp. 1-27. doi:10.1016/j.surfrep.2010.10.001

[6] S. Barbet, R. Aubry, M. A. di Forte-Poisson, J. C. Jacquet, D. Deresmes, T. Melin and D. Theron, "Surface Potential of n- and p-Type GaN Measured by Kelvin Force Microscopy," Applied Physics Letters, Vol. 93, No. 21, 2008, pp. 212107-212107-3. doi:10.1063/1.3028639

[7] Y. Rosenwaks, R. Shikler, T. Glatzel and S. Sadewasser, "Kelvin Probe Force Microscopy of Semiconductor Surface Defects," Physical Review B: Condensed Matter and Materials Physics, Vol. 70, No. 8, 2004. doi:10.1103/PhysRevB.70.085320

[8] J. Lu, L. Eng, R. Bennewitz, E. Meyer, H. J. Guntherodt, E. Delamarche and L. Scandella, "Surface Potential Studies of Self-Assembling Monolayers Using Kelvin Probe Force Microscopy," Surface and Interface Analysis, Vol. 27, No. 5-6, 1999, pp. 368-373. doi:10.1002/(SICI)1096-9918(199905/06)27:5/6<368::AI D-SIA530>3.0.CO;2-W

[9] K. J. Kwak, S. Yoda and M. Fujihira, "Observation of Stretched Single DNA Molecules by Kelvin Probe Force Microscopy," Applied Surface Science, Vol. 210, No. 1-2, 2003, pp. 73-78. doi:10.1016/S0169-4332(02)01482-4

[10] S. Kalinin and D. Bonnell, "Local Potential and Polarization Screening on Ferroelectric Surfaces," Physical Review B: Condensed Matter and Materials Physics, Vol. 63, No. 12, 2001. doi:10.1103/PhysRevB.63.125411

[11] S. V. Kalinin, C. Y. Johnson and D. A. Bonnell, "Domain Polarity and Temperature Induced Potential Inversion on the BaTiO[sub 3](100) Surface," Journal of Applied Physics, Vol. 91, No. 6, 2002, p. 3816. doi:10.1063/1.1446230

[12] V. M. Fridkin, "Ferroelectric Semiconductors," Consultants Bureau, 1980.

[13] Q. Li, Y. Liu, D. Y. Wang, R. L. Withers, Z. R. Li, H. S. Luo and Z. Xu, "Switching Spectroscopic Measurement of Surface Potentials on Ferroelectric Surfaces via an Open-Loop Kelvin Probe Force Microscopy Method," Applied Physics Letters, Vol. 101, No. 24, 2012. doi:10.1063/1.4772511

[14] K. Lau, Y. Liu, Q. Li, Z. R. Li, R. L. Withers and Z. Xu, "Domain-Selective Photochemical Reaction on Oriented Ferroelectric $\mathrm{Pb}\left(\mathrm{In}_{1 / 2} \mathrm{Nb}_{1 / 2}\right) \mathrm{O}_{3}-\mathrm{Pb}\left(\mathrm{Mg}_{1 / 3} \mathrm{Nb}_{2 / 3}\right) \mathrm{O}_{3}-\mathrm{PbTiO}_{3}$ Single Crystals," Applied Surface Science, Vol. 265, No., 2013, pp. 157-161.

[15] Y. Kim, S. Buhlmann, J. Kim, M. Park, K. No, Y. K. Kim and S. Hong, "Local Surface Potential Distribution in Oriented Ferroelectric Thin Films," Applied Physics Letters, Vol. 91, No. 5, 2007, Article ID: 052906. doi:10.1063/1.2761502

[16] Y. Kim, C. Bae, K. Ryu, H. Ko, Y. K. Kim, S. Hong and H. Shin, "Origin of Surface Potential Change during 
Ferroelectric Switching in Epitaxial PbTiO3 Thin Films Studied by Scanning Force Microscopy," Applied Physics Letters, Vol. 94, No. 3, 2009, Article ID: 032907. doi:10.1063/1.3046786

[17] Y. Kim, M. Park, S. Buhlmann, S. Hong, Y. K. Kim, H. Ko, J. Kim and K. No, "Effect of Local Surface Potential Distribution on Its Relaxation in Polycrystalline Ferroelectric Films," Journal of Applied Physics, Vol. 107, No. 5, 2010. doi:10.1063/1.3290953

[18] J. Shen, H. Z. Zeng, Z. H. Wang, S. B. Lu, H. D. Huang and J. S. Liu, "Study of Asymmetric Charge Writing on $\mathrm{Pb}(\mathrm{Zr}, \mathrm{Ti}) \mathrm{O}-3$ Thin Films by Kelvin Probe force Microscopy," Applied Surface Science, Vol. 252, No. 22, 2006, pp. 8018-8021. doi:10.1016/j.apsusc.2006.01.078

[19] H. Yamada, X. Q. Chen, T. Horiuchi, K. Matsushige, S. Watanabe, M. Kawai and P. S. Weiss, "Surface Potential of Ferroelectric Thin Films Investigated by Scanning Probe Microscopy," Journal of Vacuum Science \& Technology B, Vol. 17, No. 5, 1999, pp. 1930-1934. doi:10.1116/1.590851

[20] J. Heo, "A Study of the Ferroelectric Properties of $\mathrm{PbZr}_{0.4} \mathrm{Ti}_{0.6} \mathrm{O}_{3}$ (PZT) Grains Using Kelvin Force Microscopy Analysis," Transactions on Electrical and Electronic Materials, Vol. 11, No. 6, 2010, pp. 275-278.

\section{doi:10.4313/TEEM.2010.11.6.275}

[21] Q. Li, Y. Liu, J. Schiemer, P. Smith, Z. Li, R. L. Withers and $\mathrm{Z}$. Xu, "Fully-Inverted Piezoresponse Hysteresis Loops Mediated by Charge Injection in $\mathrm{Pb}\left(\mathrm{In}_{1 / 2} \mathrm{Nb}_{1 / 2}\right) \mathrm{O}_{3^{-}}$ $\mathrm{Pb}\left(\mathrm{Mg}_{1 / 3} \mathrm{Nb}_{2 / 3}\right) \mathrm{O}_{3}-\mathrm{PbTiO}_{3}$ Single Crystals," Applied Physics Letters, Vol. 98, No. 9, 2011, Article ID: 092908. doi:10.1063/1.3562034

[22] Y. L. Qian Li, R. L. Withers, Y. H. Wan, Z. R. Li and Z. $\mathrm{Xu}$, "Piezoresponse Force Microscopy Studies on the Domain Structures and Local Switching Behavior of $\mathrm{Pb}$ $\left(\mathrm{In}_{1 / 2} \mathrm{Nb}_{1 / 2}\right) \mathrm{O}_{3}-\mathrm{Pb}\left(\mathrm{Mg}_{1 / 3} \mathrm{Nb}_{2 / 3}\right) \mathrm{O}_{3}-\mathrm{PbTiO}_{3}$ Single Crystals," Journal of Applied Physics, Vol. 112, No. 5, 2012, Article ID: 052006

[23] A. L. Kholkin, I. K. Bdikin, V. V. Shvartsman and N. A. Pertsev, "Anomalous Polarization Inversion in Ferroelectrics via Scanning Force Microscopy," Nanotechnology, Vol. 18, No. 9, 2007. doi:10.1088/0957-4484/18/9/095502

[24] Y. J. Oh, J. H. Lee and W. Jo, "Dynamics of Space and Polarization Charges of Ferroelectric Thin Films Measured by Atomic Force Microscopy," Ultramicroscopy, Vol. 106, No. 8-9, 2006, pp. 779-784. doi:10.1016/j.ultramic.2005.12.015 